\title{
Erratum to: Mechanically controlled quantum switch defined on a curved 2DEG*
}

\section{Eur. Phys. J. B (2020) 93 : 163, https://doi.org/10.1140/epjb/e2020-10070-1}

S. Seyyare Aksu ${ }^{1}$, Oguzhan Kasikci ${ }^{1}$, and Afif Siddiki ${ }^{2, a}$

${ }^{1}$ Physics Department, Faculty of Sciences and Letters, Mimar Sinan Fine Arts University, 34380-Sisli, Istanbul, Turkey

2 Department of Electrics and Electronics, Faculty Engineering and Natural Sciences, Maltepe University, 34857 Istanbul, Turkey

Received 7 September 2020 / Accepted 7 September 2020

Published online 9 November 2020

(C) EDP Sciences / Società Italiana di Fisica / Springer-Verlag GmbH Germany, part of Springer Nature, 2020

In the original article, the first author's name was mistakenly written as S. Seyyare Aksul. It must be corrected to S. Seyyare Aksu.

The publisher apologizes for the mistake.

\footnotetext{
${ }^{\star}$ The online version of the original paper can be found at https://doi.org/10.1140/epjb/e2020-10070-1

a e-mail: afifsiddiki@gmail.com
} 